\title{
A Cluster Algorithm for Monte Carlo Simulation at Constant Pressure
}

\author{
N.G. Almarza \\ Instituto de Química-Física Rocasolano (CSIC), \\ C/ Serrano 119, E-28006 Madrid, Spain
}

(Dated: April 15, 2009)

\begin{abstract}
We propose an efficient algorithm to sample the volume in Monte Carlo simulations in the isobaric-isothermal ensemble. The method is designed to be applied in the simulation of hardcore models at high density. The algorithm is based in the generation of clusters of particles. At the volume change step, the distances between pairs of particles belonging to the same cluster do not change. This is done by rescaling the positions of the center of mass of each cluster instead of the position of each individual particle. We have tested the performance of the algorithm by simulating fluid and solid phases of hard spheres, finding that in both cases the algorithm is much more efficient than the standard procedure. Moreover, the efficiency of the method measured in terms of correlation "time" does not depend on the system size in contrast with the standard method, in which the sampling becomes rapidly inefficient as the system size increases. We have used the procedure to compute with high precision the equation of state of the face-centered cubic phase of the hard sphere system for different system sizes. Using these results we have estimated the equation of state at the thermodynamic limit. The results are compared with different equations of state proposed in the literature.
\end{abstract}




\section{INTRODUCTION}

Monte Carlo (MC) simulations in the Isobaric-Isothermal (NPT) and Grand Canonical (GC) ensembles are nowadays essential tools in the calculation of phase transitions of model systems. ${ }^{1-3}$ In systems with hard-core interactions the standard techniques ${ }^{1,2}$ of sampling the volume in the NPT simulation become inefficient for large systems at moderate or high densities. The simple scaling of the particle positions with the simulation box length(s) implies, for simple models, that the acceptance of a compression trial is limited by the minimum pair distance. As a consequence a proper sampling of the density fluctuations using NPT MC could require long simulation runs.

In some cases one can make use of simulation in Canonical or GC ensembles to avoid the problems, but there are situations like one-component phase transitions at high density or fluid phase equilibria of mixtures where the natural choice is to work with a fixed total number of particles and sampling the fluctuations of the volume, and eventually the shape, of the simulation box. ${ }^{4,5}$ It is therefore desirable to develop general algorithms to make NPT simulations of hard core systems as efficient as, for instance, those of the Lennard-Jones model.

The development of cluster flipping methods ${ }^{3,6-10}$ has made possible the study of the critical behavior of a number of simple systems considering very large system sizes. This kind of methods were firstly applied to lattice spin systems $s^{6,7,11,12}$. Further generalizations allowed their application to spin fluids, ${ }^{13}$ and fluid mixtures with symmetric interactions ${ }^{14,15}$. The construction of efficient cluster algorithms for more complicated systems is not straightforward, but some efficient methods have been developed; for instance, the so-called Geometrical Cluster algorithms ${ }^{16-19}$ have been applied to the simulation of fluid mixtures; cluster algorithms have also been used for the simulation of systems which present aggregation of particles at different length scales ${ }^{20,21}$; and finally an efficient cluster algorithm has been recently proposed to perform Monte Carlo simulations of dense systems using multiple particle moves $^{22}$.

In this paper we will present an efficient cluster $\mathrm{MC}$ algorithm to sample the volume in MC simulation of systems with hard-core interactions. The algorithm follows some rules to generate clusters of particles. Then, each of the clusters is treated like a rigid molecule in the volume sampling moves. The performance of the algorithm will be tested by simulating 
fluid and solid hard sphere (HS) systems.

The paper is organized as follows, after this introduction, in Section II we review the standard procedure of performing the volume sampling in Monte Carlo simulations, and analyze the scaling of its performance with the system size in the case of HS. In section III we present the cluster algorithm, the acceptance criteria, and some important details to consider when simulating systems with periodic boundary conditions (PBC). Section IV is devoted to the calculations carried out to test the method, including simulations of fluid and solid phases of HS at the melting pressure, and the computation of the equation of state (EOS) of a HS solid phase. Finally in section $\mathrm{V}$ we include some final remarks and the main conclusions of the paper.

\section{SIMULATIONS ON THE ISOBARIC-ISOTHERMAL ENSEMBLE}

For the sake of simplicity we will consider simple systems simulated using PBC on cubic boxes. However, a number of generalizations to confined systems, non-cubic boxes or anisotropic models are straightforward. Considering a given configuration of a simple system with volume $V$, one can reduce the position coordinates of the particles as:

$$
\alpha_{x i}=x_{i} / L ; \quad \alpha_{y i}=y_{i} / L ; \quad \alpha_{z i}=z_{i} / L
$$

where $L=V^{1 / 3}$. According to the usual formulation of the classical statistical mechanics applied to the simulation of systems with $\mathrm{PBC}^{1-3}$, the probability of a such a configuration in the NPT ensemble can be written as:

$$
P\left(\alpha^{3 N} \mid V\right) \propto V^{N} \exp \left[-\beta p V-\beta \mathcal{U}\left(\alpha^{3 N}, V\right)\right]
$$

where $\alpha^{3 N}$ represent the reduced coordinates of the $N$ particles, $p$ is the external pressure to which the system is exposed, $\beta=1 / k_{B} T$, with $T$ being the temperature and $k_{B}$ is the Boltzmann's constant, and $\mathcal{U}$ is the potential energy. In the simulations reported in this work we have made use of the probability given by Eq. (2). Nevertheless, notice that in spite of the wide use of Eq. (2) in computer simulation, recent derivations of the statistical mechanics of the constant pressure ensembles ${ }^{23-29}$ have been proposed. For the particular case of homogeneous systems with PBC, these formulations suggest that the proper probability in the NPT ensemble should be slightly modified ${ }^{26-28}$ from that given in Eq. (2), by taking 
$N-1$ (instead of $N)$ as the exponent of $V$. Nevertheless, at the thermodynamic conditions considered in this work, the influence of such a possible modification of $P\left(\alpha^{3 N} \mid V\right)$ on the simulation results is expected to be very small for most of the simulated system sizes used in the simulations. ${ }^{26,28}$

In the standard NPT MC sampling ${ }^{1}$, a trial volume $V^{\prime}$ is generated, for instance, by using

$$
V^{\prime}=V+(2 \xi-1) \Delta_{V}^{\max }
$$

where $\xi$ is a random number uniformly distributed in the range $(0,1)$, and $\Delta_{V}^{\max }$ is a fixed parameter. Given the prescription (3) and the probability in the NPT ensemble -eq. 2- the trial volume $V^{\prime}$ is accepted as the new volume of the system with probability $A\left(V^{\prime} \mid V\right)$ given by $^{1,2}$ :

$$
A\left(V^{\prime} \mid V\right)=\max \left[1, \frac{\exp \left[N \log V^{\prime}-\beta p V^{\prime}-\beta \mathcal{U}\left(\alpha^{3 N}, V^{\prime}\right)\right]}{\exp \left[N \log V-\beta p V-\beta \mathcal{U}\left(\alpha^{3 N}, V\right)\right]}\right]
$$

In order to consider the ability of a certain algorithm to sample the volume fluctuations it is useful to consider the average of the absolute values of the volume change in the corresponding sampling steps, $\langle|\Delta V|>$ (notice that rejected attempts also contribute to the average with $\Delta V=0$ ). Then, one can consider, in principle, that the better algorithm will produce the larger values of $\langle|\Delta V|>$. Of course, the ability of the MC calculation to sample the volume fluctuations depends not only on the volume sampling algorithm, but also in the efficiency of the other types of motions included in the simulation procedure. Nevertheless, in this contribution we will analyze how to improve the volume sampling, which can help to build up more efficient algorithms for systems where the difficulty of obtaining appropriate values of $\langle|\Delta V|>$ is the bottleneck of the simulation. As an example we have chosen HS systems.

\section{A. Hard spheres}

In the case of HS one can make a theoretical analysis of the efficiency of standard NPT codes by using approximate EOSs and some basic statistical mechanics. The pressure of the system in the Canonical ensemble can be computed as ${ }^{2,30}$

$$
\beta p=\frac{N}{V}-\lim _{\Delta V \rightarrow 0^{+}}\left[\frac{1}{\Delta V} \log \langle A(V-\Delta V \mid V)\rangle\right] .
$$


where $\langle A(V-\Delta V \mid V)\rangle$ is the fraction of the acceptable configurations $\alpha^{N}$ for the volume $V$ that are also acceptable for the volume $V^{\prime}=V-\Delta V$. Defining $\beta p_{e x}=\beta p-\rho$, with $\rho \equiv N / V$, we can write for $\Delta V<<V$;

$$
\begin{gathered}
\beta p_{e x} \simeq-\frac{\log \langle A(V-|\Delta V| \mid V)\rangle}{\Delta V} ; \\
\langle A(V-\Delta V \mid V)\rangle \simeq \exp \left[-\beta p_{e x} \Delta V\right] .
\end{gathered}
$$

Using Eq. (7) it is possible to estimate the fraction of accepted volume change trials and the value of $\langle|\Delta V|>$ for a HS simulation in the NPT ensemble. The basic result for our purposes is that the results for $\left\langle|\Delta V|>\right.$, for a given value of $\Delta_{V}^{\max }$, will depend on the pressure but will be practically independent of $N$. Considering that the fluctuations of the volume for an homogeneous system scale as:

$$
(\delta V)^{2}=<V^{2}>-<V>^{2} \propto N
$$

the expected number of cycles required to sample the density fluctuations of the system in a MC simulation using the standard algorithm will scale as $N_{\text {cycles }} \propto N$.

\section{A CLUSTER ALGORITHM TO SAMPLE THE VOLUME}

The basic problem of the volume sampling in hard-core systems is that the value of $<|\Delta V|>$ is strongly conditioned by the presence of some pairs of particles at distances very close to the HS diameter, $\sigma$. In order to avoid (to some extent) the overlap of close pairs of particles we can define bonds between them. Considering these bonds one can build up clusters that will behave as rigid pseudo-molecules when reducing the volume, i.e., the absolute distances between particles belonging to the same cluster will stay fixed in the volume sampling step. We expect that this strategy will allow us to increase the parameter $\Delta_{V}^{\max }$ and subsequently the value of $\langle|\Delta V|>$. This kind of procedures has been applied in the simulation of surfactant assemblies ${ }^{20}$ and ionic fluids ${ }^{21}$. In these two cases the clusters were built using deterministic topological criteria.

\section{A. Cluster construction and trial configuration}

In order to generate clusters we firstly generate bonds between pairs of particles. The probability of generating a bond between two particles depends on their distance. We have 
taken a bonding probability, $b(r)$, given by:

$$
b(r)=\left\{\begin{array}{ll}
1-\left(\frac{r-\sigma}{\sigma \delta}\right)^{2} & ; \sigma \leq r \leq(1+\delta) \sigma \\
0 & ; r>(1+\delta) \sigma
\end{array} .\right.
$$

With this prescription, pairs of particles separated by a short distance $r$, with $\sigma<r<\sigma+\delta \sigma$, have some probability of being bonded (larger as $r$ approaches $\sigma$ ). Notice that other choices for $b(r)$ are possible. The basic reason to choose a functional form like that given in Eq. (9) is that it ensures a high probability of bonding pairs of particles at distances very close to the HS diameter. In addition we think that this prescription is convenient due to its simplicity

and its short range. Nevertheless, no systematic analysis of the numerical efficiency of different equations for $b(r)$ has been performed. The bonds are implemented using Eq. (9) and a pseudo-random number generator. This is carried out as follows, for each pair of particles lying at a distance $r_{i j}<(1+\delta) \sigma$, we generate a random number, $\xi$, uniformly distributed in the range $(0,1)$; a bond between the particles is then created if $\xi<b\left(r_{i j}\right)$. These bonds will allow us to define clusters of particles.

The volume sampling step is carried out generating a test volume $V^{\prime}$ using the usual procedures -for instance Eq.(3)-, and the particle positions in the trial configuration are built up by conserving the reduced coordinates of the center of mass of each cluster, and the absolute distances between pairs of particles belonging to the same cluster. Notice that, in the particular case of absence of bonds each particle forms an independent cluster, and the resulting configuration is equivalent to that generated by the standard procedure.

\section{B. Acceptance rules}

Once the trial configuration has been generated, one has to apply the adequate acceptance criteria that ensure that the sampling of the system configurations corresponds to the selected weighting function. Obviously, for the particular case of particles with hard core interactions, the existence of overlaps in the trial configuration leads to a direct rejection. In order to explain the acceptance rules in the NPT ensemble we can consider the clusters as rigid molecules, then we have to take into account the probabilities of building up the particular cluster realization in both the original and the trial configuration. According to the detailed balance condition ${ }^{1,2}$, we can write:

$$
P\left(V \mid \mathcal{X}_{c}\right) \omega_{c}\left(\mathcal{X}_{c} \mid \mathbf{X}\right) \omega_{v}\left(V^{\prime} \mid V\right) A\left(V^{\prime} \mid V\right)=P\left(V^{\prime} \mid \mathcal{X}_{c}\right) \omega_{c}\left(\mathcal{X}_{c} \mid \mathbf{X}^{\prime}\right) \omega_{v}\left(V \mid V^{\prime}\right) A\left(V \mid V^{\prime}\right)
$$


where $\omega_{c}\left(\mathcal{X}_{c} \mid \mathbf{X}\right)$ is the probability of generating a particular cluster realization $\mathcal{X}_{c}$ (represented by the internal coordinates and reduced position coordinates of the centers of mass of the clusters) from a given set of reduced coordinates of the particles and volume (represented as $\mathbf{X}$ or $\left.\mathbf{X}^{\prime}\right) ; \omega_{v}\left(V_{a} \mid V_{b}\right)$ is the probability of choosing $V_{a}$ as trial volume when the system has a volume $V_{b}$. Considering the system as composed of rigid molecules, we get:

$$
P\left(V \mid \mathcal{X}_{c}\right) \propto V^{N_{c}} \exp \left[-\beta p V-\beta \mathcal{U}\left(\mathcal{X}_{c}, V\right)\right]
$$

where $N_{c}$ is the number of clusters ${ }^{31}$. Notice that $N_{c}$ does not depend on the individual configurations $\mathbf{X}$, and $\mathbf{X}^{\prime}$ considered independently; in fact $N_{c}$ is actually a property of the cluster structure that makes possible the transformation between the two configurations. Given the method of choosing the trial volume -Eq $(3)-$ we have $\omega_{v}\left(V^{\prime} \mid V\right)=\omega_{v}\left(V \mid V^{\prime}\right)$. Therefore the acceptance criteria will fulfill:

$$
\frac{A\left(V^{\prime} \mid V ; \mathcal{X}_{c}\right)}{A\left(V \mid V^{\prime} ; \mathcal{X}_{c}\right)}=\frac{\left(V^{\prime}\right)^{N_{c}} \exp \left[-\beta p V^{\prime}-\beta \mathcal{U}\left(\mathcal{X}_{c}, V^{\prime}\right)\right]}{V^{N_{c}} \exp \left[-\beta p V-\beta \mathcal{U}\left(\mathcal{X}_{c}, V\right)\right]} \frac{\omega_{c}\left(\mathcal{X}_{c} \mid \mathbf{X}^{\prime}\right)}{\omega_{c}\left(\mathcal{X}_{c} \mid \mathbf{X}\right)}
$$

This acceptance ratio is similar to that of the standard procedures except for two details; the number of clusters $N_{c}$ enters in the formula (instead of $N$ ); and the presence in Eq. (12) of the ratio of the probabilities of generating the cluster structure. The computation of this ratio can be done following the same strategies well known in many clusters algorithms, and can be found elsewhere ${ }^{6,7,10,22}$. The final result is:

$$
\frac{\omega\left(\mathcal{X}_{c} \mid \mathbf{X}^{\prime}\right)}{\omega\left(\mathcal{X}_{c} \mid \mathbf{X}\right)}=\prod_{[i j]} \frac{1-b\left(r_{i j}^{\prime}\right)}{1-b\left(r_{i j}\right)}
$$

Where $[i j]$ represents pairs of particles belonging to different clusters. In practice, the number of pairs to be considered, can be reduced by taking into account only pairs with $r_{i j}$ (or) $r_{i j}^{\prime}<\sigma+\sigma \delta$.

\section{Effect of the periodic boundary conditions}

There are two main issues that have to be considered when applying the algorithm described above to the simulation of systems using PBC. The first one is the possibility that, at least, one cluster percolates through the system. This means that every particle in such a cluster is linked via a line of bonds to some of its periodic images, if this happens the cluster becomes of infinite length (via replication through the $\mathrm{PBC}$ ) and obviously it is not 
possible to modify the size of the simulation box while keeping constant the intra-cluster distances. Therefore, if cluster percolation occurs, the change of volume is not possible and we can consider the corresponding MC step as rejected.

On the other hand, even if no percolation occurs, we can find the case in which two particles belonging to the same cluster, that are far apart considering the intra-cluster distance, can be very close if their distance is computed via minimum image convention, and eventually being at a distance less than $(1+\delta) \sigma$. Such distances are actually defined over two particles belonging to different images of the same cluster; and are expected to change when rescaling the system size, and therefore have to be included in the computation of (13).

\section{CALCULATIONS}

We have tested the the performance of the proposed cluster algorithm using HS systems as benchmark. We have run simulations using both the standard $(\delta=0)$ and the cluster method. Firstly we run a number of test simulations to find optimal values of the sampling parameters for the cluster algorithm $\left(\Delta_{V}^{\max }\right.$ and $\left.\delta\right)$. The optimal parameters were chosen as those that maximize the average values of $|\Delta v| \equiv|\Delta V| / N$, obtained in the volume change attempts. As expected, such parameters also provide the smallest error bars for the mean values of the volume per particle and the density for a fixed simulation length. Notice, however that for the standard procedure, alternative criteria to define optimal parameters in terms of CPU time instead of simulation length could be considered due to the lower computational cost of rejected MC moves. ${ }^{2}$ In the case of the cluster algorithm we find that the best values for $\delta$ seem to be independent of the system size, whereas the optimum value for $\Delta_{v}^{\max }=\Delta_{V}^{\max } / N$ scales as $\Delta_{v}^{\max } \propto N^{-1 / 2}$.

The translational attempts were performed using the standard procedure by generating the new position with uniform probability in a cube of length $l=2 \Delta_{x}^{\max }$ centered at the current position.

The analysis of the efficiency of the simulation was performed as in previous work ${ }^{22}$ using the mean density as the target property. In this way we can estimate the correlation length of each simulation by comparing the statistics of the block averages with the fluctuations of the corresponding quantity. The analysis has been carried out for fluid and solid phases of 
HS at the melting pressure.

\section{A. Simulations at the melting transition pressure}

We have simulated fluid and crystal phases of HS at reduced pressure $\beta p \sigma^{3}=11.54$, which corresponds to a recent and precise estimation of the fluid-solid transition of $\mathrm{HS}^{32}$. For the fluid phase we took $\Delta_{x}^{\max }=0.10 \sigma$, which produces about a $22 \%$ of acceptance in the translational attempts, whereas for the solid phase $\Delta_{x}^{\max } / \sigma=0.0866$ was chosen with about $27 \%$ of accepted attempts. The parameters to sample the volume were taken as follows. For the standard method we used:

$$
\Delta_{v}^{\max } \simeq \frac{1.8 \sigma^{3}}{(Z-1) N}
$$

where the compressibility factor, $Z \equiv p v / k_{B} T$, was taken from approximate EOSs; in particular we used the Carnahan-Starling $\operatorname{EOS}^{33}$ for the fluid, and a simple EOS for the solid that will be detailed later in the paper. The results given in Eq. (14) can be derived by computing the expected mean value of $|\Delta V|$ as a function of $\Delta_{v}^{\max }$ by considering that the acceptance probability of a given (virtual) trial volume change in the canonical ensemble is related with the pressure of the system ${ }^{2}$. For the cluster method, after a number of tests using systems with small numbers of particles, we chose $\delta \simeq 0.025$ and $\Delta_{v}^{\max } \simeq 0.086 / \sqrt{N}$ for both phases. The fraction of accepted trials in volume sampling was close to $50 \%$ for both methods, whereas for the cluster method the average of the number of bonds in one configuration was, for both fluid and solid phases, $\left\langle N_{b}\right\rangle / N \simeq 0.53$.

The main results are presented in Tables I and II. The results for the fluid phase agree within statistical uncertainty with those given by Kolafa et al. ${ }^{34}$ EOS, whereas the results for the solid phase are very close to those given by Speedy's ${ }^{35}$ EOS. From these tables it is clear that the cluster algorithm is much more efficient than the standard one for all the system sizes considered. Moreover, as the system size increases the standard procedure suffers of a dramatic increase of the correlation time, growing as $L_{c o r r}(N) \sim N$, whereas the same measure of the efficiency is not system size dependent for the cluster algorithm. As can be seen in the tables for systems of about 864 particles the cluster algorithm is already a hundred times faster than the standard one.

Of course, the cluster algorithm is a bit more expensive in terms of CPU per simulation cycle (with a cycle consists of $N$ translational trial moves and one volume change attempt). 
Nevertheless this difference (the cluster algorithm required less than double the time of the standard algorithm for the same number of cycles) becomes negligible considering the dramatic increasing of sampling efficiency.

\section{B. Equation of state of the solid phase}

Given the good precision of the results we have used the cluster algorithm to compute the EOS of the face-centered cubic solid of HS. In order to attain this goal we have carried out MC simulations for several pressures using different numbers of particles, $N=4 i^{3}$, with $i=3,4,5, \cdots, 12$. According to theoretical ${ }^{36}$ and simulation ${ }^{35,37-39}$ results the EOS of the solid phase can be written using expansions as:

$$
v^{*}=v_{0}^{*}+\sum_{i=1}^{n} a_{i} y^{i} .
$$

where $v^{*}$ is the reduced volume per particle $v^{*} \equiv V /\left(N \sigma^{3}\right), v_{0}^{*}$ is the limit of $v^{*}$ at high pressure $v_{0}^{*} \equiv 1 / \sqrt{2} ; y=1 /\left(\beta p \sigma^{3}\right)$; and $a_{1}=3$ in the thermodynamic limit. In order to select the maximum displacement parameters of the simulation we can take the approximate equation:

$$
v^{*}(y) \simeq v_{1}^{*}(y)=v_{0}^{*}+3 y
$$

Accordingly we have taken $\Delta_{x}^{\max }$ and $\delta$ to be approximately proportional to $\left(r_{1}-\sigma\right)$, with $r_{1}$ being the distance between nearest neighbors in the perfect lattice. The fluctuations of the volume per particle will be $\left\langle\left(v^{*}\right)^{2}>-<v^{*}>^{2} \simeq 3 y^{2} / N\right.$, which invites to consider $\Delta_{v}^{\max } \propto y / \sqrt{N}$. In practice our choice of parameters was $\Delta_{x}^{\max } / \sigma=1.0 y, \Delta_{v}^{\max } / \sigma^{3}=y / \sqrt{N}$ and $\delta / \sigma=0.30 y$. The prefactors were chosen attending to the optimization carried out previously for $\beta p \sigma^{3}=11.54$.

Simulations were carried out for 21 values of $y$ in the range $0<y \leq 0.09[(y=k \times 0.005$ with $k=1,2,3, \cdots, 18)$; and $y=0.001,0.002$, and, 1/11.54]. The correlation times for the different values of $y$ and $N$ are similar to those presented in Table II and hardly depend on $y$. The average of the number of bonds was found, as expected, proportional to $N$, and showed some dependence with $y$, ranging from $\left\langle N_{b}\right\rangle / N \simeq 0.44$ for low values of $y$ to $<N_{b}>/ N \simeq 0.53$ for $y=0.09$. In order to analyze the results we found convenient to express the EOS in terms of the function $a(y, N) \equiv \beta p\left(<v(y, N)>-v_{0}\right)$, where $<v(y, N)>$ is the average of the volume per particle at the corresponding values of $y$ and $N$. The function 
$a(y, N)$ exhibits values close to the expected value $a(y \rightarrow 0, N \rightarrow \infty)=3$ in all the range of the stable crystal region. The error bars of $a(y, N)$ for a given system size, using the parameter selection described above, and equal simulation lengths hardly depend on $y$. In tables III and IV we present the simulation results.

We have fitted the simulation results for each system size as:

$$
a(y, N)=\sum_{i=0}^{n} a_{i+1}(N) y^{i} .
$$

We found that using $n=5$ allows a good fitting of the results for all the system sizes considered. In addition, for each value of $y$ we have estimated the value $a(y)=\lim _{N \rightarrow \infty} a(y, N)$. For fixed pressure, we found that the results as a function of $N$ can be represented as:

$$
a(y, N)=a(y)+\sum_{i=1}^{m} b_{i}(y) \frac{1}{N^{i}}
$$

where, for each value of $y, m$ is chosen to be either $m=1$ or $m=2$ using a chi-square test ${ }^{40}$. The values $a(y)$ can then be used to extract an EOS for the system in the thermodynamic limit. The coefficients $a_{i}(N)$ together with those of the fitting of the extrapolated values $a(y)$ are presented in table $\mathrm{V}$. The fitting of the extrapolated values $a(y)$ provides the result $a_{1}=3.00001(11)$, which is consistent with the expected behavior ${ }^{35,37-39}$. Therefore we computed our final estimation of the EOS in the thermodynamic limit by fixing $a_{1} \equiv 3$ as a constraint. The result for the EOS with this consideration reads:

$$
\beta p\left(v-v_{0}\right)=3-1.807846 y+11.56350 y^{2}+141.6000 y^{3}-2609.260 y^{4}+19328.09 y^{5} .
$$

The EOS given in Eq. (19) produces results very close to those coming from Speedy's $\operatorname{EOS}^{35}$ for the range of studied pressures $(0<y \leq 0.09)$, or $\beta p \sigma^{3}>11.11$. The largest deviations, either in terms of $a(y)$ or in the compressibility factor, $Z=\beta p v$, occur precisely close to the lower bound of the pressure. Speedy's EOS can be written as:

$$
a(v)=\beta p\left(v-v_{0}\right)=3-c_{1}\left(1-v_{0} / v\right) \frac{v_{0} / v-c_{2}}{v_{0} / v-c_{3}} .
$$

Using our extrapolated data $a(y)$, we have estimated the values of the parameters $c_{i}$ to be $c_{1}=0.5914, c_{2}=0.7079$, and $c_{3}=0.6022$ which are not far from those reported by Speedy which are respectively $0.5921,0.7072$, and $0.601^{35}$.

The results for $a(v)$ or $Z$ from both sets of parameters are quite similar, with the maximum difference in the range $v_{0}^{*} \leq v \leq 0.975$ being less than $8 \times 10^{-4}$, which corresponds to 
differences in the density for a given pressure of less than $10^{-4}$. The differences are within the error bars of Speedy's results We have estimated the error bar of our extrapolated $a(y)$ to be $\Delta a(y)<2 \times 10^{-4}$.

The differences between our results and other EOSs found in the literature ${ }^{37-39}$ are larger, which is not surprising since the simulation data used in their fittings have much larger uncertainties than both Speedy's and our results, and some of the data come from simulation of relatively small system sizes.

\section{The fluid phase}

In addition to the simulations for the fluid phase presented in Table I we carried out additional cluster $\mathrm{MC}$ simulations at $\beta p \sigma^{3}=1.0$, and 2.0. In both cases we found consistently that a good choice of the volume change parameters can be found by switching $\delta$ to produce $<N_{b}>\simeq N / 2$ or $<N_{c}>\simeq N / 2$; and selecting $\Delta_{v}^{\max }$ to get about $50 \%$ of accepted volume change trials. As in the results for the fluid at $\beta p \sigma^{3}=11.54$ the results of the average of the reduce density for systems with $N \geq 864$ do not show significant system size dependence and agree within statistical uncertainty $\left(\Delta \rho \leq 10^{-4}\right.$ in these cases) with the results extracted from the EOS of Kolafa et $a l .^{34}$.

\section{FINAL REMARKS AND CONCLUSIONS}

Some small caution has to be taken when using the cluster algorithm presented in this paper. Firstly one has to take some care in the way of managing the products of the non-bonding probabilities appearing in Eq. (13). For large systems, these products can eventually exhaust the capability of the computer to store small numbers, therefore it is advisable to bypass this possible problem by working in terms of the logarithms of the different quantities. The second caution is related with the possibility of bond percolation. In principle this should not be a problem if one has performed a number of short tests before choosing adequate parameters to run the simulations. Nevertheless, if by chance one uses as starting configuration of the simulation at a given pressure, a configuration with an inappropriate high density, it can happen that all the bond realizations built to perform the volume sampling percolate and do not allow any volume change. 
To conclude, in this paper we have presented an algorithm to perform NPT simulation of hard core models. We have simulated HS systems to check the performance of the algorithm, and it has been shown that the efficiency can be increased in orders of magnitude with respect to the standard methods, specially for large systems. We have obtained very precise results that allowed us to check, first the correctness of the procedure, and second the quality of some of the most precise EOSs for HS found in the literature. In particular we have confirmed the extreme precision of the results provided by the EOS proposed by Kolafa et $a l .{ }^{34}$ for the HS fluid phase. In addition, for the face-centered cubic solid phase our results indicate that the EOS by Speedy ${ }^{35}$ is very precise, but can be improved further by an slight refinement of the adjustable parameters.

The cluster algorithms presented in this paper are expected to enhance spectacularly the efficiency of NPT simulations of many systems containing hard core interactions, in particular, we have made some preliminary checks that confirm that this is the case for systems as hard-core Yukawa, hard sphere + square well models, and athermal models which form liquid crystals.

A simple adaptation of the ideas behind the cluster algorithm can also be useful to build up more efficient algorithms to simulate systems that can form aggregates like micelles.

\section{Acknowledgments}

The author gratefully acknowledges the support from the Dirección General de Investigación Científica y Técnica under Grant No. MAT2007-65711-C04-04 and from the Dirección General de Universidades e Investigación de la Comunidad de Madrid under Grant No. S0505/ESP/0299 and Program MOSSNOHO-CM.

1 M.P. Allen and D.J. Tildesley, Computer Simulation of Liquids, (Clarendon Press, Oxford, 1986).

2 D. Frenkel and B. Smit, Understanding Molecular Simulation, (Academic Press, 2002).

3 D.P. Landau, and K. Binder, A guide to Monte Carlo Simulations in Statistical Physics, 2n edition, Cambridge University Press, 2005.

4 N.G. Almarza, E.Lomba, C. Martin, and A. Gallardo, J. Chem. Phys. 129234504 (2008). 
5 G. Ganzenmller, and P.J. Camp, J. Chem. Phys. 127, 154504 (2007).

6 R.H. Swendsen, and J.S. Wang, Phys. Rev. Lett. 58, 86 (1987).

7 U. Wolff, Phys. Rev. Lett. 62, 361(1989).

8 J. Machta, Y.S. Choi, A. Lucke, T. Schweizer, and. L.V. Chayes, Phys. Rev. Lett. 75, 2792 (1995).

9 Y. Tomita, and Y. Okabe, Phys. Rev. Lett. 86, 572 (2001).

10 K. Binder and D. W. Hermann, Monte Carlo Simulation in Statistical Physics, 3rd edition, Springer Series in Solid-State Sciences Vol.80 , (Springer, Heidelberg, 1997)

11 H. Kunz, G. Zumbach, Phys. Rev. B 46, 662 (1992).

12 N.V. Priezjev and R.A. Pelcovits, Phys. Rev. E 63, 062702 (2001).

13 E. Lomba, C. Martin, and N.G. Almarza, Mol. Phys. 101, 1667 (2003).

14 G. Johnson, H. Gould, J. Machta, and L.K. Chayes, Phys. Rev. Lett. 79, 2612 (1997).

15 R. Sun, H. Gould, J. Machta, and L.W. Chayes, Phys. Rev. E 62, 2226 (2000).

16 C. Dress and W. Krauth, J. Phys. A 28, L597 (1995).

17 J. Liu and E. Luijten, Phys. Rev. Lett. 92, 035504 (2004).

18 J. Liu and E. Luijten, Phys. Rev. E 71, 066701 (2005).

19 A. Buhot, J. Chem. Phys. 122, 024105 (2005).

20 D. Wu, D. Chandler, and B. Smit, J. Phys. Chem. 96, 4077 (1992).

21 G. Orkoulas, and A. Z. Panagiotopoulos, J. Chem. Phys. 101, 1452 (1994).

22 N.G. Almarza and E. Lomba, J. Chem. Phys. 127, 084116 (2007).

23 G.J.M. Koper, and H. Reiss, J. Phys. Chem. 100, 422 (1996).

24 D.S. Corti, and G. Soto-Campos, J. Chem. Phys. 108, 7959 (1998).

25 D.S. Corti, Phys. Rev E 64, 016128 (2001).

26 D.S. Corti, Mol. Phys. 100, 1887 (2002).

27 K. Han, and H.S. Son, J. Chem. Phys. 115, 7793 (2001).

28 P. Attard, J. Chem. Phys. 103, 9884 (1995).

29 M.J. Uline, and D.S. Corti, J. Chem. Phys. 129, 014107 (2008).

30 E. de Miguel, and G. Jackson, J. Chem. Phys. 125, 125, 164109 (2006).

31 The exponent of $V$ in Eq. (11) should be $N_{c}-1$ instead of $N_{c}$ if we consider the alternative formulation of the NPT ensemble mentioned in Section II.

32 C. Vega, and E.G. Noya, J. Chem. Phys. 127, 154113 (2007). 
33 N.F. Carnahan, and K.E. Starling, J. Chem. Phys., 51, 635 (1969).

34 J. Kolafa, S. Labík, and A. Malijevský, Phys. Chem. Chem. Phys. 6, 2335 (2004).

35 R.J. Speedy, J. Phys.: Condens. Matter 10, 4387 (1998).

36 M. Baus, and C.F. Tejero, Equilibrium Statistical Physics, Phases of Matter and Phase Transitions, (Springer, 2008).

37 K.R. Hall, J. Chem. Phys., 57, 2252 (1972)

38 B. J. Alder, W. G. Hoover, and D. A. Young, J. Chem. Phys. 49, 3688 (1968)

39 R. Hoste and W. van Dael, J. Chem. Soc., Faraday Trans. 2, 80, 477 (1984).

40 W.H. Press et al., Numerical Recipes. The Art of Scientific Computing, 3rd edition (Cambridge University Press, Cambridge, 2007). 
TABLE I: Simulation results for the fluid phase of hard spheres, at reduced pressure $\beta p \sigma^{3}=11.54$. $\mathrm{NC}$ is the number of cycles used in the calculation of the properties, $L_{c o r r}$ represents the number of MC cycles required to get effectively independent configurations of the system. Error bars are given between parentheses in units of the last figure of the property and correspond to a confidence level of about $95 \%$. The results for the largest systems agree with the EOS of Kolafa et al ${ }^{34}$, which renders $\rho^{*} \simeq 0.93871$ for the same pressure, whereas the result for the Carnahan-Starling ${ }^{33}$ EOS is $\rho^{*}=0.93898$.

\begin{tabular}{l|lll|lll}
\hline \hline & Cluster & & & \multicolumn{3}{l}{ Standard } \\
\hline$N$ & $\mathrm{NC} / 10^{6}$ & $\log _{10} \mathrm{~L}_{\text {corr }}$ & $<\rho \sigma^{3}>$ & $\mathrm{NC} / 10^{6}$ & $\log _{10} \mathrm{~L}_{\text {corr }}$ & $<\rho \sigma^{3}>$ \\
\hline 256 & 28.672 & 2.5 & $0.93932(6)$ & 28.672 & 4.1 & $0.9393(4)$ \\
500 & 28.672 & 2.6 & $0.93860(4)$ & 28.672 & 4.4 & $0.9385(4)$ \\
864 & 14.336 & 2.5 & $0.93865(5)$ & 28.672 & 4.6 & $0.9388(4)$ \\
1372 & 7.168 & 2.5 & $0.93871(5)$ & 14.336 & $\sim 4.8$ & $0.9386(5)$ \\
2048 & 7.168 & 2.6 & $0.93873(5)$ & & & \\
2916 & 7.168 & 2.6 & $0.93873(4)$ & & & \\
4000 & 7.168 & 2.6 & $0.93871(3)$ & & & \\
5324 & 7.168 & 2.5 & $0.93871(3)$ & & & \\
6912 & 7.168 & 2.6 & $0.93871(2)$ & & & \\
\hline
\end{tabular}


TABLE II: Simulation results for the face-centered cubic solid phase of hard spheres, at reduced pressure $\beta p \sigma^{3}=11.54$, and their extrapolation to the thermodynamic limit. See Table I for explanation of symbols and error bars. The results for large system sizes are very close to that from Speedy's equation of state ${ }^{35}$, which renders $\rho \sigma^{3} \simeq 1.03694$

\begin{tabular}{r|ccc|ccc} 
& Cluster & & \multicolumn{3}{|l}{ Standard } \\
\hline$N$ & $\mathrm{NC} / 10^{6}$ & $\log _{10} \mathrm{~L}_{\text {corr }}$ & $<\rho \sigma^{3}>$ & $\mathrm{NC} / 10^{6}$ & $\log _{10} \mathrm{~L}_{\text {corr }}$ & $<\rho \sigma^{3}>$ \\
\hline 108 & 28.672 & 2.2 & $1.03934(8)$ & 57.344 & 3.7 & $1.0396(3)$ \\
256 & 28.672 & 2.3 & $1.03789(6)$ & 57.344 & 4.1 & $1.0380(3)$ \\
500 & 28.672 & 2.2 & $1.03740(4)$ & 57.344 & 4.4 & $1.0375(3)$ \\
864 & 14.336 & 2.3 & $1.03717(4)$ & 57.344 & 4.6 & $1.0373(3)$ \\
1372 & 7.168 & 2.3 & $1.03710(5)$ & & & \\
2048 & 7.168 & 2.3 & $1.03701(4)$ & & & \\
2916 & 7.168 & 2.3 & $1.03695(4)$ & & & \\
4000 & 7.168 & 2.3 & $1.03697(3)$ & & & \\
5324 & 7.168 & 2.2 & $1.03692(3)$ & & & \\
6912 & 7.168 & 2.2 & $1.03692(2)$ & & & \\
$\infty$ & & & $1.03688(2)$ & & & \\
\hline
\end{tabular}


TABLE III: Results for $\beta p\left(<v>-v_{0}\right)$ for different system sizes $\mathrm{N}=108,256,500,864,1372$; and reduced pressures. The last row indicates the statistical uncertainty (corresponding to a confidence level of about $95 \%$ ) of the data of each column.

\begin{tabular}{c|llllll}
\hline \hline $1 /\left(\beta p \sigma^{3}\right)$ & $\mathrm{N}=108$ & $\mathrm{~N}=256$ & $\mathrm{~N}=500$ & $\mathrm{~N}=864$ & $\mathrm{~N}=1372$ \\
\hline 0.00100 & 2.97994 & 2.98975 & 2.99421 & 2.99557 & 2.99686 \\
0.00200 & 2.97746 & 2.98871 & 2.99261 & 2.99380 & 2.99483 \\
0.00500 & 2.97317 & 2.98348 & 2.98727 & 2.98899 & 2.98966 \\
0.01000 & 2.96500 & 2.97558 & 2.97913 & 2.98075 & 2.98181 \\
0.01500 & 2.95766 & 2.96821 & 2.97199 & 2.97336 & 2.97448 \\
0.02000 & 2.95122 & 2.96186 & 2.96546 & 2.96699 & 2.96788 \\
0.02500 & 2.94534 & 2.95609 & 2.95955 & 2.96151 & 2.96238 \\
0.03000 & 2.94061 & 2.95085 & 2.95471 & 2.95626 & 2.95719 \\
0.03500 & 2.93649 & 2.94650 & 2.95006 & 2.95187 & 2.95235 \\
0.04000 & 2.93192 & 2.94290 & 2.94663 & 2.94841 & 2.94897 \\
0.04500 & 2.92995 & 2.94028 & 2.94357 & 2.94572 & 2.94661 \\
0.05000 & 2.92723 & 2.93894 & 2.94225 & 2.94373 & 2.94468 \\
0.05500 & 2.92607 & 2.93713 & 2.94094 & 2.94304 & 2.94399 \\
0.06000 & 2.92630 & 2.93723 & 2.94142 & 2.94277 & 2.94343 \\
0.06500 & 2.92678 & 2.93769 & 2.94230 & 2.94365 & 2.94483 \\
0.07000 & 2.92865 & 2.94066 & 2.94448 & 2.94628 & 2.94713 \\
0.07500 & 2.93203 & 2.94395 & 2.94805 & 2.95005 & 2.95098 \\
0.08000 & 2.93747 & 2.94929 & 2.95362 & 2.95571 & 2.95670 \\
0.08500 & 2.94375 & 2.95687 & 2.96169 & 2.96361 & 2.96454 \\
0.08666 & 2.94579 & 2.95980 & 2.96455 & 2.96688 & 2.96739 \\
0.09000 & 2.95296 & 2.96732 & 2.97201 & 2.97396 & 2.97550 \\
\hline \hline$\Delta a$ & 0.0008 & 0.0006 & 0.0005 & 0.0005 & 0.0005 \\
\hline \hline
\end{tabular}


TABLE IV: Results for $\beta p\left(<v>-v_{0}\right)$ for different system sizes $(\mathrm{N}=2048,2916,4000,5324,6912$, and the extrapolated value at $N=\infty$ ) and reduced pressures. The last row indicates the statistical uncertainty of the data (corresponding to a confidence level of about $95 \%$ ) of each column.

\begin{tabular}{c|lllllll}
\hline \hline $1 /\left(\beta p \sigma^{3}\right)$ & $\mathrm{N}=2048$ & $\mathrm{~N}=2916$ & $\mathrm{~N}=4000$ & $\mathrm{~N}=5324$ & $\mathrm{~N}=6912$ & Extrap. \\
\hline 0.00100 & 2.99713 & 2.99785 & 2.99775 & 2.99782 & 2.99787 & 2.99828 \\
0.00200 & 2.99545 & 2.99555 & 2.99585 & 2.99616 & 2.99621 & 2.99643 \\
0.00500 & 2.99021 & 2.99039 & 2.99084 & 2.99095 & 2.99090 & 2.99122 \\
0.01000 & 2.98235 & 2.98268 & 2.98270 & 2.98262 & 2.98292 & 2.98316 \\
0.01500 & 2.97500 & 2.97510 & 2.97538 & 2.97530 & 2.97564 & 2.97584 \\
0.02000 & 2.96833 & 2.96858 & 2.96884 & 2.96872 & 2.96914 & 2.96928 \\
0.02500 & 2.96268 & 2.96265 & 2.96286 & 2.96318 & 2.96314 & 2.96344 \\
0.03000 & 2.95776 & 2.95765 & 2.95788 & 2.95818 & 2.95814 & 2.95846 \\
0.03500 & 2.95307 & 2.95315 & 2.95343 & 2.95365 & 2.95365 & 2.95392 \\
0.04000 & 2.94972 & 2.94976 & 2.95022 & 2.95013 & 2.95022 & 2.95054 \\
0.04500 & 2.94735 & 2.94750 & 2.94734 & 2.94759 & 2.94752 & 2.94790 \\
0.05000 & 2.94501 & 2.94527 & 2.94524 & 2.94559 & 2.94566 & 2.94583 \\
0.05500 & 2.94430 & 2.94411 & 2.94442 & 2.94461 & 2.94468 & 2.94498 \\
0.06000 & 2.94378 & 2.94437 & 2.94436 & 2.94462 & 2.94459 & 2.94487 \\
0.06500 & 2.94488 & 2.94555 & 2.94573 & 2.94574 & 2.94582 & 2.94616 \\
0.07000 & 2.94755 & 2.94804 & 2.94815 & 2.94808 & 2.94833 & 2.94858 \\
0.07500 & 2.95138 & 2.95160 & 2.95194 & 2.95199 & 2.95219 & 2.95247 \\
0.08000 & 2.95698 & 2.95765 & 2.95769 & 2.95789 & 2.95814 & 2.95839 \\
0.08500 & 2.96543 & 2.96513 & 2.96554 & 2.96588 & 2.96610 & 2.96634 \\
0.08666 & 2.96832 & 2.96885 & 2.96869 & 2.96915 & 2.96912 & 2.96950 \\
0.09000 & 2.97630 & 2.97628 & 2.97644 & 2.97684 & 2.97677 & 2.97721 \\
\hline$\Delta a$ & 0.0004 & 0.0004 & 0.0003 & 0.0003 & 0.0002 & 0.0002 \\
\hline \hline
\end{tabular}


TABLE V: Results for the fitting of the MC simulation results of the face-centered cubic solid phase for different system sizes. In the first two rows are presented the results of the fitting of the values extrapolated to the thermodynamic limit, with and without the constraint $a_{1}=3$.

\begin{tabular}{c|cccccc}
\hline \hline$N$ & $a_{1}$ & $a_{2}$ & $a_{3}$ & $a_{4}$ & $a_{5}$ & $a_{6}$ \\
\hline $\operatorname{EXT}\left(a_{1}=3\right)$ & 3 & -1.807846 & 11.56350 & 141.6000 & -2609.260 & 19328.09 \\
$\mathrm{EXT}$ & 3.000011 & -1.810289 & 11.71624 & 137.6795 & -2565.141 & 19147.50 \\
6912 & 2.999648 & -1.785399 & 10.40428 & 161.2374 & -2708.428 & 19240.01 \\
5324 & 2.999734 & -1.847007 & 13.86740 & 94.2559 & -2228.810 & 18362.19 \\
4000 & 2.999439 & -1.782818 & 9.84831 & 184.8341 & -3060.398 & 20927.82 \\
2916 & 2.999328 & -1.796961 & 9.87670 & 205.1110 & -3481.881 & 23289.57 \\
2048 & 2.998921 & -1.784469 & 10.67893 & 169.7043 & -3110.876 & 22320.72 \\
1372 & 2.998394 & -1.747346 & 7.05421 & 276.3954 & -4294.302 & 26629.29 \\
864 & 2.997368 & -1.782900 & 11.58475 & 125.0455 & -2323.746 & 17714.23 \\
500 & 2.995964 & -1.762799 & 8.02275 & 247.6868 & -3905.027 & 24603.63 \\
256 & 2.991778 & -1.695544 & 5.30957 & 304.3872 & -4549.552 & 27369.28 \\
108 & 2.981524 & -1.799479 & 14.27503 & 14.4766 & -683.191 & 8744.69 \\
\hline \hline
\end{tabular}

